\title{
Clinical Trial Site
}

National Cancer Institute

\section{Source}

National Cancer Institute. Clinical Trial Site. NCI Thesaurus. Code C85838.

Any healthcare organization, institution, facility or provider directly involved in conducting or facilitating a particular clinical trial. 